\title{
Moments and Reduction for Symplectic Groupoids
}

\author{
By \\ Kentaro MIKAMI* and Alan WEINSTEIN**
}

\section{\$ 0. Introduction}

A hamiltonian action of a Lie group $G$ on a symplectic manifold $M$ is generated by a momentum map $J: M \rightarrow \mathrm{g}^{*}$ which is equivariant with respect to the coadjoint representation. The reduction procedure of Meyer [14] and Marsden and Weinstein [13] consists of forming the quotient $M_{\mu}=J^{-1}(\mu) / G_{\mu}$, where $\mu$ is an element of $\mathrm{g}^{*}$ and $G_{\mu}$ is its coadjoint isotropy group.

In recent years (see [6], for example) a property of $J$ already known to Lie [9] has been recognized as essential: $J$ is a Poisson map from $M$ to $\mathrm{g}^{*}$ with its Lie-Poisson structure. This suggests the problem of replacing $\mathrm{g}^{*}$ by an arbitrary Poisson manifold $P$, but the question immediately arises as to what object will play the role of the group $G$. This object having just been identified by Karasev [7] and one of us [3] [20] as a symplectic groupoid, the purpose of the present paper is to extend the reduction procedure to symplectic groupoid actions.

An important stimulus for our work has been the reduction theory for Poisson Lie group actions developed in [16]. Using Drinfel'd's notion of Poisson Lie group [4], Semenov-Tian-Shansky explained the hamiltonian behavior of the dressing transformations which arise from the inverse-scattering approach to completely integrable systems. A new theory was necessary because dressing transformations do not preserve the Poisson structure of the spaces on which they act. In a sequel to this paper, we hope to study how Poisson Lie groups and

Communicated by H. Araki, July 20, 1987.

* Department of Mathematics, Akita University, Akita 010, Japan.

** Department of Mathematics, University of California, Berkeley, CA 94720, U. S. A. 
their actions can be lifted to symplectic double groupoids and symplectic groupoid actions, so that at least part of the reduction theory in [16] can be understood in our terms.

Our paper is organized as follows. In $\S 1$, we review the basic properties of groupoids, both as algebraic and symplectic objects. The remaining two sections can be read independently. In $\S 2$, we discuss actions of groups as automorphisms on symplectic groupoids, observing that in many cases the groupoid structure implies that the action has a momentum mapping of a very special form. $\$ 3$ contains the discussion of reduction. We observe that this construction, usually thought of as "symplectic", has a counterpart in the purely algebraic setting of groupoid actions [2] [5].

Most of the work reported here was completed before V. P. Maslov kindly called to our attention Karasev's paper [7], in which some of our results are anticipated. In particular, we wish to acknowledge that symplectic groupoids were already studied in [7], where they were called "Lie pseudogroups". Although this name effectively identifies these objects as generalized Lie groups, it carries other connotations related to the general theory of pseudogroups, so we have chosen to keep our terminology.

\section{Acknowledgements}

K. Mikami would like to express his sincere thanks to the University of California, Berkeley, and especially to Prof. and Mrs. Shoshichi Kobayashi for their kindness and hospitality while he was in Berkeley. His research was partially supported by Grant-in-Aid for Scientific Research (No. 62540009), Ministry of Education, Science and Culture, Japan.

A. Weinstein's research was partially supported by NSF grant DMS-84-03201 and DOE contract ATO 381-ER 12097. He would also like to thank the University of Tokyo for hospitality during the period when this paper was completed. 


\section{\$1. Basic Properties of Symplectic Groupoids}

In this section, we review for completeness some of the material from [3] [7] and [20]. (Also see [22].) Briefly, a groupoid is a category whose morphisms are all invertible, and a symplectic groupoid has a symplectic structure for which the graph of the product map is lagrangian.

Definition $1_{0} \mathbb{1}_{0}$ A groupoid $\Gamma$ over (a set) $\Gamma_{0}$ is a pair $\left(\Gamma, \Gamma_{0}\right)$ equipped with:

(i) surjections $\alpha, \beta: \Gamma \rightarrow \Gamma_{0}$ (called the source and target maps respectively);

(ii) $m: \Gamma_{2} \rightarrow \Gamma$ (multiplication), where $\Gamma_{2}:=\{(x, y) \in \Gamma \times \Gamma \mid \beta(x)=$ $\alpha(y)\}$; each pair $(x, y)$ in $\Gamma_{2}$ is said to be composable;

(iii) an injection $\varepsilon: \Gamma_{0} \rightarrow \Gamma$ (identities);

(iv) $\ell: \Gamma \rightarrow \Gamma$ (inversion).

These maps must satisfy:

(1) (associative law) $m(m(x, y), z)=m(x, m(y, z))$ (if one is defined, so is the other);

(2) (identities) for each $x,(\varepsilon(\alpha(x)), x) \in \Gamma_{2},(x, \varepsilon(\beta(x))) \in \Gamma_{2}$ and $m(\varepsilon(\alpha(x)), x)=m(x, \varepsilon(\beta(x)))=x$;

(3) (inverses) for each $x,(x, \imath(x)) \in \Gamma_{2,}(\ell(x), x) \in \Gamma_{2}, m(x, \iota(x))$ $=\varepsilon(\alpha(x))$, and $m(\ell(x), x)=\varepsilon(\beta(x))$.

We summarize some properties of these mappings, obtained directly from the definition.

Proposition 1.2. (i) $\alpha \circ \varepsilon=\beta \circ \varepsilon=i d_{\Gamma_{0}}$.

(ii) $\quad \alpha(m(x, y))=\alpha(x)$ and $\beta(m(x, y))=\beta(y)$.

(iii) For each $u \in \Gamma_{0}, m(\varepsilon(u), \varepsilon(u))=\varepsilon(u)$.

(iv) (cancellation) If $m\left(x, y_{1}\right)=m\left(x, y_{2}\right)$ or $m\left(y_{1}, z\right)=m\left(y_{2}, z\right)$, then $y_{1}=y_{2}$.

(v) $\quad \mathrm{e} \bullet \mathrm{\ell}=i d_{\Gamma}$.

(vi) $\alpha \circ \iota=\beta$ and $\beta \circ \iota=\alpha$.

With the same notation as in Definition 1.1, we state: 
Definition 1.3. A groupoid $\Gamma$ over $\Gamma_{0}$ is a Lie groupoid if $\Gamma$ and $\Gamma_{0}$ are differentiable manifolds, $\alpha$ and $\beta$ are differentiable submersions so that $\Gamma_{2}$ is a differentiable submanifold of the product manifold $\Gamma \times \Gamma, \varepsilon$ is a differentiable embedding, and $m$ and $\iota$ are differentiable. We call $\Gamma \alpha-[$ simply] connected if the fibres of $\alpha$ (and hence of $\beta$ ) are [simply] connected. (By definition, simple connectivity includes connectivity.)

Definition 1.4. A symplectic groupoid $\Gamma$ over $\Gamma_{0}$ is a symplectic manifold $(\Gamma, \Omega)$ with a Lie groupoid structure over $\Gamma_{0}$ such that the graph $\mathscr{M}:=\left\{(x, y, m(x, y)) \in \Gamma \times \Gamma \times \Gamma \mid(x, y) \in \Gamma_{2}\right\}$ of the groupoid multiplication $m$ is a lagrangian submanifold of

$$
(\Gamma, \Omega) \times(\Gamma, \Omega) \times(\Gamma,-\Omega) .
$$

Denote the $\alpha$ - and $\beta$-fibres through $x$ by $\alpha_{x}$ and $\beta_{x}$; i. e., $\alpha_{x}$ := $\alpha^{-1}(\alpha(x))$ and $\beta_{x}:=\beta^{-1}(\beta(x))$ respectively. Directly from Definition 1. 4, we can prove:

Proposition 1.5. (i) $\varepsilon\left(\Gamma_{0}\right)$ is a lagrangian submanifold of $(\Gamma, \Omega)$.

(ii) The inversion 6 is an anti-symplectic diffeomorphism of $(\Gamma, \Omega)$.

(iii) For each $x \in \Gamma$, the tangent spaces of $\alpha_{x}$ and $\beta_{x}$ are symplectically orthogonal at $x$.

Proof. (i) It follows from $(x, \varepsilon \circ \beta(x), x) \in \mathscr{M}$ that $(\delta x, T(\varepsilon \circ \beta)(\delta x), \delta x)$ $\in T \mathscr{M}$ and $\left(\delta^{\prime} x, T(\varepsilon \circ \beta)\left(\delta^{\prime} x\right), \delta^{\prime} x\right) \in T \mathscr{M}$, where $\delta x$ and $\delta^{\prime} x$ are tangent vectors to $\Gamma$ at $x$. Since $\mathscr{M}$ is lagrangian, we have

$$
\begin{aligned}
0 & =\Omega\left(\delta x, \delta^{\prime} x\right)+\Omega\left(T(\varepsilon \circ \beta)(\delta x), T(\varepsilon \circ \beta)\left(\delta^{\prime} x\right)\right)-\Omega\left(\delta x, \delta^{\prime} x\right) \\
& =\Omega\left(T(\varepsilon \circ \beta)(\delta x), T(\varepsilon \circ \beta)\left(\delta^{\prime} x\right)\right) .
\end{aligned}
$$

Letting $\delta x$ and $\delta^{\prime} x$ be tangent to $\varepsilon\left(\Gamma_{0}\right)$ and using Proposition 1.2 (i), we conclude that $\varepsilon\left(\Gamma_{0}\right)$ is an isotropic submanifold. Now $3 \operatorname{dim} \Gamma=$ $2 \operatorname{dim} \mathscr{M}=2\left\{\operatorname{dim} \Gamma+\operatorname{dim} \beta_{x}\right\}=2\{\operatorname{dim} \Gamma+(\operatorname{dim} \Gamma-\operatorname{rank} \beta)\}=2(2 \operatorname{dim} \Gamma$ $\left.-\operatorname{dim} \Gamma_{0}\right)=4 \operatorname{dim} \Gamma-2 \operatorname{dim} \Gamma_{0}$, i. e, $\quad \operatorname{dim} \Gamma=2 \operatorname{dim} \Gamma_{0}=2 \operatorname{dim} \varepsilon\left(\Gamma_{0}\right)$. Thus, $\varepsilon\left(\Gamma_{0}\right)$ is a lagrangian submanifold of $\Gamma$.

(ii) Similarly, since $(\ell(x), x, \varepsilon \circ \beta(x)) \in \mathscr{M}$ implies that $\left(T_{l}(\delta x), \delta x, T(\varepsilon \circ \beta)(\delta x)\right) \in T \mathscr{M}$ and $\left(T_{l}\left(\delta^{\prime} x\right), \delta^{\prime} x, T(\varepsilon \circ \beta)\left(\delta^{\prime} x\right)\right) \in T \mathscr{M}$, we have 


$$
\begin{aligned}
0 & =\Omega\left(T_{\ell}(\delta x), T_{\ell}\left(\delta^{\prime} x\right)\right)+\Omega\left(\delta x, \delta^{\prime} x\right)-\Omega\left(T(\varepsilon \circ \beta)(\delta x), T(\varepsilon \circ \beta)\left(\delta^{\prime} x\right)\right) \\
& =\Omega\left(T_{\ell}(\delta x), T_{l}\left(\delta^{\prime} x\right)\right)+\Omega\left(\delta x, \delta^{\prime} x\right) .
\end{aligned}
$$

(iii) Let $\delta x$ and $\delta^{\prime} x$ be arbitrary tangent vectors at $x$ to $\alpha_{x}$ and $\beta_{x}$ respectively, and let $c(t)$ be a curve in $\alpha_{x}$ with $\dot{c}(0)=\delta x$. Since $(\varepsilon \circ \alpha(x), c(t), c(t)) \in \mathscr{M}$, we have $(0, \delta x, \delta x) \in T \mathscr{M}$. Likewise, we have $\left(\delta^{\prime} x, 0, \delta^{\prime} x\right) \in T \mathscr{M}$. Since $\mathscr{M}$ is isotropic, $\Omega\left(\delta x, \delta^{\prime} x\right)$ must be zero. 圈

Theorem 1.6。 (i) $T_{x} \alpha_{x}=\left\{X_{\beta^{*} f}(x) \mid f \in C^{\infty}\left(\Gamma_{0}\right)\right\}$ and $T_{x} \beta_{x}=\left\{X_{\alpha^{n} f}(x) \mid f\right.$ $\left.\in C^{\infty}\left(\Gamma_{0}\right)\right\}$, where the notation $X_{F}$ means the hamiltonian vector field of $F$.

(ii) $\alpha^{*} C^{\infty}\left(\Gamma_{0}\right)$ and $\beta^{*} C^{\infty}\left(\Gamma_{0}\right)$ commute with one another in the Lie algebra $C^{\infty}(\Gamma)$ with respect to the Poisson bracket induced from the symplectic structure $\Omega$. If $\Gamma$ is $\alpha$-connected, then $\alpha^{*} C^{\infty}\left(\Gamma_{0}\right)$ and $\beta^{*} C^{\infty}\left(\Gamma_{0}\right)$ are the full centralizers of one another.

(iii) There exists a unique Poisson structure on $\Gamma_{0}$ such that $\alpha$ and $\beta$ are Poisson and anti-Poisson mappings.

To prove Theorem 1.6, we need some preparation. For two subsets $A$ and $B$ of $\Gamma$, let $A \cdot B$ denote the set $\{m(x, y) \mid x \in A, y \in B$, $\left.(x, y) \in \Gamma_{2}\right\}$. We say that a submanifold $\Sigma$ of $\Gamma$ is horizontal ${ }^{1)}$ if $\alpha_{\mid \Sigma}$ and $\beta_{\mid \Sigma}$ are embeddings onto open subsets of $\Gamma_{0}$ A horizontal submanifold $\Sigma$ defines two local transformations on $\Gamma$ as follows:

$\varkappa_{\Sigma}: \beta^{-1}(\alpha(\Sigma)) \rightarrow \beta^{-1}(\beta(\Sigma)) ; x \mapsto\{x\} \cdot \Sigma$ (right translation), and

$\ell_{\Sigma}: \alpha^{-1}(\beta(\Sigma)) \rightarrow \alpha^{-1}(\alpha(\Sigma)) ; x \mapsto \Sigma \circ\{x\}$ (left translation).

We leave to the reader the exercise of proving the following elementary results.

Proposition 1.7. (i) $\alpha \circ x_{\Sigma}=\alpha, \beta \circ \iota_{\Sigma}=\beta$.

(ii) If $\Sigma$ and $\Sigma^{\prime}$ are horizontal, then $\Sigma \cdot \Sigma^{\prime}$ and $\Sigma^{\prime} \cdot \Sigma$ are horizontal, $x_{\Sigma}^{\circ} x_{\Sigma^{\prime}}=x_{\Sigma^{\prime} \cdot \Sigma}$, and $\ell_{\Sigma} \circ \ell_{\Sigma^{\prime}}=\ell_{\Sigma \cdot \Sigma^{\prime}}$ （Equality of two local transformations means in part that their domains, possibly empty, are equal.)

(iii) If $\Sigma$ is horizontal, then $\Sigma^{-1}$ is also horizontal and

$$
\left(x_{\Sigma}\right)^{-1}=x_{\Sigma^{-1}},\left(\ell_{\Sigma}\right)^{-1}=\ell_{\Sigma^{-1}}
$$

where $\Sigma^{-1}:=\ell(\Sigma)$. (Here, when we write $x^{-1}$, we mean $\operatorname{domain}\left(x^{-1}\right)=$

1) A horizontal manifold is called a "bissection" in [3]. 
range $(x)$ and vice versa.)

(iv) $x_{\Sigma}^{\circ} \ell_{\Sigma}^{\prime}=\ell_{\Sigma}, 0 x_{\Sigma}$.

Lemma 1.8. (i) If a horizontal submanifold $\Sigma$ is lagrangian, then $\varkappa_{\Sigma}$ and $\ell_{\Sigma}$ are local symplectic diffeomorphisms.

(ii) Conversely, if $x_{\Sigma}$ or $l_{\Sigma}$ is symplectic, then $\Sigma$ is a lagrangian submanifold.

(iii) $\mathscr{R}:=\left\{\varkappa_{\Sigma} \mid \Sigma\right.$ horizontal lagrangian submanifold $\}$ and $\mathscr{L}:=\left\{\ell_{\Sigma} \mid \Sigma\right.$ horizontal lagrangian submanifold\} form commuting pseudogroups of symplectic transformations of $\Gamma$ whose orbits are the $\alpha$ - and $\beta$-fibres respectively.

Proof. Fix an arbitrary point $x_{0} \in \beta^{-1}(\alpha(\Sigma))$. There is a unique local section $\sigma$ of $\alpha$ around $\beta\left(x_{0}\right)$ such that $\operatorname{Im}(\sigma) \subset \Sigma$ and $\beta \circ \sigma$ is a diffeomorphism. For each $x$ near $x_{0}, x_{\Sigma}(x)=m(x, \sigma(\beta(x))$, i. e, $\left(x, \sigma(\beta(x)), \varkappa_{\Sigma}(x)\right) \in \mathscr{M}$ and so $\left(\delta x, T(\sigma \circ \beta) \delta x, T_{\varkappa_{\Sigma}}(\delta x)\right),\left(\delta^{\prime} x, T(\sigma \circ \beta) \delta^{\prime} x\right.$, $\left.T_{\varkappa_{\Sigma}}\left(\delta^{\prime} x\right)\right) \in T \mathscr{M}$. Since $\mathscr{M}$ is lagrangian, we have

$$
0=\Omega\left(\delta x, \delta^{\prime} x\right)+\Omega\left(T(\sigma \circ \beta) \delta x, T(\sigma \circ \beta) \delta^{\prime} x\right)-\Omega\left(T_{\varkappa_{\Sigma}}(\delta x), T_{\varkappa_{\Sigma}}\left(\delta^{\prime} x\right)\right) .
$$

(i) If $\Sigma$ and so $\operatorname{Im}(\sigma)$ is lagrangian, then

$$
0=\Omega\left(\delta x, \delta^{\prime} x\right)-\Omega\left(T_{\varkappa_{\Sigma}}(\delta x), T_{\varkappa_{\Sigma}}\left(\delta^{\prime} x\right)\right) .
$$

Therefore, $x_{\Sigma}^{*} \Omega=\Omega$. Similarly, $\ell_{\Sigma}^{*} \Omega=\Omega$.

(ii) Conversely, assume that $x_{\Sigma}$ is symplectic. Then the equation above implies

$$
\Omega\left(T(\sigma \circ \beta) \delta x, T(\sigma \circ \beta) \delta^{\prime} x\right)=0,
$$

i. e., $\operatorname{Im}(\sigma)$ is lagrangian. A similar argument applies to $\zeta_{\Sigma}$.

(iii) It follows from (i), (ii), and Proposition 1.7 that $\mathscr{R}$ and $\mathscr{L}$ form pseudogroups of symplectic transformations of $\Gamma$, that $\mathscr{R}$ and $\mathscr{L}$ commute, and that $\mathscr{R}[\mathscr{L}]$ leaves each $\alpha$ - $[\beta-]$ fibre invariant. Given any elements $y_{0}$ and $y_{1}$ in $\alpha_{x}$, choose horizontal lagrangian submanifolds $\Sigma_{i}$ for $i=0,1$, such that $\Sigma_{i} \cap \alpha_{x}=\left\{y_{i}\right\}$ for each $i$. Then we have $x_{\Sigma_{1}} \circ\left(x_{\Sigma_{0}}\right)^{-1}\left(y_{0}\right)=x_{\Sigma_{0}-1 \cdot \Sigma_{1}}\left(y_{0}\right)=y_{1}$. Thus, each $\alpha$-fibre is an $\mathscr{R}$-orbit. Likewise, each $\beta$-fibre is an $\mathscr{L}$-orbit.

Proof of Theorem 1.6. (i) By Lemma 1.8 (iii), for each $x \in \Gamma$, the $\mathscr{R}$-orbit $\left\{x_{\Sigma}(x) \mid \Sigma\right.$ horizontal lagrangian $\}$ equals the $\alpha$-fibre $\alpha_{x}$. For an arbitrary tangent vector $\delta x$ to $\alpha_{x}$ at $x$, take a curve $c(t) \in \alpha_{x}$ such that $c(0)=x$ and $\dot{c}(0)=\delta x$. For each $t$, choose a horizontal 
lagrangian submanifold depending smoothly on $t$, say $\Sigma_{t}$, such that $\Sigma_{t} \cap \alpha_{x}=\{c(t)\}$. Then we have $x_{\Sigma_{t}}{ }^{\circ}\left(x_{\Sigma_{0}}\right)^{-1}(x)=x_{\Sigma_{0}^{-1} \cdot \Sigma_{t}}(x)=c(t)$. The family $\left\{\varphi_{t}\right\}=\left\{x_{\Sigma_{t}} \circ\left(x_{\Sigma_{0}}\right)^{-1}\right\}_{t}$ defines a local vector field $Y=\frac{d}{d t} \varphi_{\left.t\right|_{t=0}}$ around $x$ with $Y(x)=\delta x$. Since $\left\{\varphi_{t}\right\}$ is a family of local symplectic diffeomorphisms, there is a local hamiltonian $F$ such that $Y=X_{F}$. Since $Y$ is tangent to $\alpha$-fibres, it follows from Proposition 1.5 (iii) that $\left.0=\Omega\left(Y, T_{y} \beta_{y}\right)=T_{y} \beta_{y}\right\rfloor d F$ for $y$ near $x$, i. e., $F$ is constant along $\beta$-fibres. Therefore, $F=\beta^{*} f$ near $x$ for some $f \in C^{\infty}\left(\Gamma_{0}\right)$, and $\delta x=$ $X_{\beta^{*} f}(x)$. By exchanging the roles of $\alpha$ and $\beta$ and right and left translations, we get the second half of assertion (i).

(ii) From Proposition 1.5 (iii), it follows that

$$
\left\{\alpha^{*} C^{\infty}\left(\Gamma_{0}\right), \beta^{*} C^{\infty}\left(\Gamma_{0}\right)\right\}=0 \text { 。 }
$$

Now let $F \in C^{\infty}\left(\Gamma_{0}\right)$ satisfy $\left\{F, \beta^{*} C^{\infty}\left(\Gamma_{0}\right)\right\}=0$. (i) yields that $F$ is locally constant along $\alpha$-fibres. Therefore, $F=\alpha^{*} f$ for some $f \in C^{\infty}\left(\Gamma_{0}\right)$ if the $\alpha$-fibres are connected.

(iii) By Lemma 1.8 (iii), $\alpha^{*}\left(C^{\infty}\left(\Gamma_{0}\right)\right)$ consists of the $\mathscr{R}$-invariant functions on $\Gamma$ and so it is closed under the bracket $\{$,$\} ; this$ induces a bracket $\{,\}_{0}$ on $\Gamma_{0}$ for which $\alpha$ is a Poisson map. Since $\beta=\alpha \circ \ell$ and $\iota$ is antisymplectic, $\beta$ is anti-Poisson.

Hereafter for simplicity we usually denote $m(x, y)$ by $x \circ y, \quad \ell(x)$ by $x^{-1},(\Gamma, \Omega) \times(\Gamma, \Omega) \times(\Gamma,-\Omega)$ by $\Gamma \oplus \Gamma \ominus \Gamma$, and its symplectic structure by $\Omega \oplus \Omega \ominus \Omega$. Note that the equation $x \cdot y=z$ means that $x$ and $y$ are composable and that the product $x \cdot y$ is equal to $z_{\text {. Some- }}$ times we identify $\Gamma_{0}$ with the submanifold $\varepsilon\left(\Gamma_{0}\right)$ of $\Gamma$.

\section{§2. Group Actions on Symplectic Groupoids}

In this section, we present some simple examples of symplectic groupoids in the course of studying momentum mappings.

Let $\pi(M)$ be the fundamental groupoid over the topological space $M$, i. e., $\pi(M):=\bigcup_{x, y} \mathscr{C}_{x, y} / \sim$, where $\mathscr{C}_{x, y}:=$ continuous $\sigma:[0,1] \rightarrow M \mid \sigma(0)$ $=x, \sigma(1)=y\}$ and " $\sim$ " is the homotopy equivalence relation. We define $\alpha([\sigma]):=\sigma(0), \beta([\sigma]):=\sigma(1), \varepsilon(x):=$ the homotopy class of the constant path at $x$, and multiplication $[\sigma] \circ[\tau]:=[\sigma \circ \tau]$, where $\sigma \circ \tau$ is the usual concatenation of curves $\sigma$ and $\tau$ such that $(\sigma \circ \tau)(0)=\sigma(0)$, 
$(\sigma \cdot \tau)(1)=\tau(1) . \quad \alpha \times \beta: \pi(M) \rightarrow M \times M$ is a groupoid homomorphism, where $M \times M$ is the coarse groupoid over $M$ (cf. Example 2. 2 below). Since $\alpha \times \beta$ is a covering map, if $M$ is a differentiable manifold, then $\pi(M)$ has a Lie groupoid structure such that $\alpha \times \beta$ is a differentiable covering map. Now assume that $M$ is a symplectic manifold with a symplectic structure $\omega$. Then $\pi(M)$ is a symplectic groupoid with the symplectic structure $\Omega:=(\alpha \times \beta) *(\omega \ominus \omega)$; the induced Poisson structure on $M$ is just the usual bracket associated with $\omega$.

Theorem 2.1. Let a Lie group $G$ act on a symplectic manifold $(M, \omega)$. The natural lift of $G$ to the fundamental groupoid $\pi(M)$ over $M$ always has an equivariant momentum mapping $J$ which is a groupoid homomorphism in the sense that $J([\sigma] \cdot[\tau])=J([\sigma])+J([\tau])$ whenever $[\sigma] \cdot[\tau]$ is defined.

Proof. Define $J: \pi(M) \rightarrow \mathrm{g}^{*}$ by $\left.\langle J([\sigma]), \xi\rangle:=-\int_{\sigma} \xi_{M}\right\rfloor \omega$ for $[\sigma] \epsilon$ $\pi(M), \xi \in g$, where $g$ is the Lie algebra of $G, g^{*}$ is the dual of $g$, and $\xi_{M}$ is the vector field on $M$ defined by the action of $\xi_{\text {。 }}$ This is welldefined on homotopy (in fact, homology) classes of paths. Clearly $J([\sigma] \cdot[\tau])=J([\sigma])+J([\tau])$.

To see that $J$ is coadjoint equivariant, we note that for each $a \in G$,

$$
\begin{aligned}
\langle J(a \cdot[\sigma]), \xi\rangle: & \left.=-\int_{a \cdot \sigma} \xi_{M}\right\rfloor \omega \\
& =\int_{0}^{1} \omega\left(a_{M}(\sigma(t))\left(\xi_{M}, T\left(a_{M}\right) \dot{\sigma}(t)\right) d t\right. \\
& =\int_{0}^{1} \omega(\sigma(t))\left(T\left(a_{M}^{-1}\right) \xi_{M}, \dot{\sigma}(t)\right) d t \\
& =\int_{0}^{1} \omega(\sigma(t))\left(\left(A d\left(a^{-1}\right) \xi\right)_{M}, \dot{\sigma}(t)\right) d t \\
& \left.=\int_{\sigma}\left(A d\left(a^{-1}\right) \xi\right)_{M}\right\rfloor \omega \\
& =\left\langle J([\sigma]), A d\left(a^{-1}\right) \xi\right\rangle \\
& =\left\langle A d\left(a^{-1}\right)^{*} J([\sigma]), \xi\right\rangle .
\end{aligned}
$$

Finally, we prove that $J$ is a momentum mapping. Take arbitrary $[\sigma] \in \pi(M)$ and $V \in T_{[\sigma]} \pi(M)$. Then, there is a variation $\Sigma:[0,1]$ $\times(-\varepsilon, \varepsilon) \rightarrow M$ of $\sigma$ such that $V(t)=T \Sigma(\partial / \partial s)(t, 0)$, where $s$ is the variable in $(-\varepsilon, \varepsilon)$. Denote $T \Sigma(\partial / \partial t)(t, s), T \Sigma(\partial / \partial s)(t, s)$ by $V_{1}(t, s)$, $V_{2}(t, s)$ respectively. Now 


$$
\begin{aligned}
-V\rfloor d \hat{J}(\xi) & \left.=\left.\frac{\partial}{\partial s}\right|_{s=0} \hat{J}(\xi)(\Sigma(\circ, s))=\left.\frac{\partial}{\partial s}\right|_{s=0} \int_{\Sigma(\cdot, s)} \xi_{M}\right\rfloor \omega \\
& =\left.\frac{\partial}{\partial s}\right|_{s=0} \int_{0}^{1} \omega\left(\xi_{M}, T \Sigma(\partial / \partial t)\right) d t=\left.\int_{0}^{1} \mathfrak{L}_{V_{2}}\left\{\omega\left(\xi_{M}, V_{1}\right)\right\}\right|_{s=0} d t \\
& =\int_{0}^{1}\left\{\left(\Omega_{V_{2}} \omega\right)\left(\xi_{M}, V_{1}\right)+\omega\left(\left[V_{2}, \xi_{M}\right], V_{1}\right)\right. \\
& \left.+\omega\left(\xi_{M},\left[V_{2}, V_{1}\right]\right)\right\}\left.\right|_{s=0} d t \\
& \left.=\int_{0}^{1}\left\{d\left(V_{2}\right\rfloor \omega\right)\left(\xi_{M}, V_{1}\right)+\omega\left(\left[V_{2}, \xi_{M}\right], V_{1}\right)\right\}\left.\right|_{s=0} d t \\
& =\int_{0}^{1}\left\{\xi_{M}\right\rfloor d\left\{\omega\left(V_{2}, V_{1}\right)-V_{1}\right\rfloor d\left\{\omega\left(V_{2}, \xi_{M}\right)\right\} \\
& =\int_{0}^{1}\left\{\omega\left(\left[\xi_{M}, V_{2}\right], V_{1}\right)+\omega\left(V_{2},\left[\xi_{M}, V_{1}\right]\right)\right. \\
& \left.\quad-V_{1}\right\rfloor d\left\{\omega\left(V_{2}, \xi_{M}\right)\right\}-\omega\left(V_{2},\left[\xi_{M}, V_{1}\right]\right) \\
\left.\quad+\omega\left(\left[V_{2}, \xi_{M}\right], V_{1}\right)\right\}\left.\right|_{s=0} d t & \left.-\int_{0}^{1} V_{1}(t, 0)\right\rfloor d\left\{\omega\left(V_{2}(t, 0), \xi_{M}\right)\right\} d t \\
= & \omega\left(V(0), \xi_{M}(\sigma(0))\right)-\omega\left(V(1), \xi_{M}(\sigma(1))\right) .
\end{aligned}
$$

On the other hand, since $\alpha \times \beta(a \cdot[\sigma])=(a \circ \sigma(0), a \circ \sigma(1))$ and so $(T(\alpha \times \beta)) \xi_{\pi(M)}([\sigma])=\left(\xi_{M}(\sigma(0)), \xi_{M}(\sigma(1))\right)$, we find

$$
\begin{aligned}
\left.V\rfloor\left(\xi_{\pi(M)}\right\rfloor \Omega\right) & =\Omega\left(\xi_{\pi(M)}, V\right) \\
& =\omega\left(\xi_{M}(\sigma(0)), V_{1}(0,0)\right)-\omega\left(\xi_{M}(\sigma(1)), V_{2}(1,0)\right) .
\end{aligned}
$$

Therefore, $\left.d \hat{J}(\xi)=\xi_{\pi(M)}\right\rfloor \Omega$ 。

Although symplectic reduction can be carried out for momentum mappings which are not coadjoint-equivariant (see [10], for instance), the equivariant case is much simpler. Given the theorem above, then, it is natural to ask the following question: Let a Lie group $G$ act on a symplectic groupoid $\Gamma$ by symplectic groupoid automorphisms. Does $G$ always have a coadjoint equivariant momentum mapping which is a groupoid homomorphism? We show in the next example that the answer to existence may be no, and then we prove a positive result with an extra hypothesis.

Example 2.2. Let $(M, \omega)$ be a symplectic manifold and let $G$ act on $M$ as symplectic automorphisms. Let $\Gamma$ be the coarse groupoid over $M, \mathrm{i}_{\mathrm{o}} \mathrm{e}, \Gamma:=M \times M, \Gamma:=M, \alpha(p, q):=p, \beta(p, q):=q, \varepsilon(p):=(p, p), \quad(p, q)$ 
- $(q, r):=(p, r),(p, q)^{-1}:=(q, p)$, and $\Omega:=\omega \ominus \omega . G$ acts on $\Gamma$ diagonally as symplectic groupoid automorphisms. It is easily seen that, if the action of $G$ on $M$ does not admit a momentum mapping, neither does the action of $G$ on $M \times M$. In particular, we may let $M$ and $G$ be the standard 2-torus with the action defined by addition.

Theorem 2.3. Let the Lie group $G$ act by automorphisms on the connected and $\alpha$-connected symplectic groupoid $\Gamma$. If the action of $G$ has any momentum mapping at all, then it has a coadjoint equivariant momentum mapping $J: \Gamma \rightarrow \mathrm{g}^{*}$ with $J(x \cdot y)=J(x)+J(y)$.

Proof. Since the action of $G$ preserves the multiplication of the groupoid $\Gamma$, it leaves $\Gamma_{0}$ invariant and commutes with $\alpha$ and $\beta_{0}$ In particular, each generating vector field $\xi_{\Gamma}$ for $\xi \in \mathrm{g}$ is tangent to $\Gamma_{0}$. Let $J_{1}$ be a momentum mapping for $G_{0}$. Since $\Gamma_{0}$ is lagrangian and $\xi_{\Gamma}$ is tangent to $\Gamma_{0}, \hat{J}_{1}(\xi)$ is locally constant on $\Gamma_{0}$ for each $\xi \in \mathrm{g}$. Since $\Gamma$ is connected, so is $\Gamma_{0}$, and so $\hat{J}_{1}(\xi)$ is constant on $\Gamma_{0}$. Therefore, if we define $\hat{J}(\xi)$ by $\hat{J}_{1}(\xi)-\hat{J}_{1}(\xi)\left(\Gamma_{0}\right)$, then $J: \Gamma \rightarrow \mathrm{g}^{*}$ is a momentum mapping which satisfies $J=0$ on $\Gamma_{0}$.

From the general theory of momentum mappings ([1] [18]), $J \circ a_{\Gamma}-a_{\mathrm{g}^{*}} \circ J$ is a locally constant map on $\Gamma$ for each $a \in G$, where $a_{\Gamma}$ is the corresponding symplectic automorphism on $\Gamma$ and $a_{\mathrm{g}^{*}}=\operatorname{Ad}\left(a^{-1}\right)^{*}$ (coadjoint action). Since $J$ vanishes on $\Gamma_{0}$ and $G$ leaves $\Gamma_{0}$ invariant, $J \circ a_{\Gamma}-a_{\mathrm{g}^{\circ}} \circ J$ vanishes for all $a \in G$, and so $J$ is coadjoint equivariant.

$J$ defines a map $K: \mathscr{M} \rightarrow \mathrm{g}^{*}$ by $K(x, y, x \cdot y):=J(x)+J(y)-J(x \cdot y)$. For each tangent vector $V \in T \mathscr{M}$, we have

$$
\begin{aligned}
\langle V\rfloor d K, \xi\rangle & \left.\left.\left.=V_{1}\right\rfloor d \hat{J}(\xi)+V_{2}\right\rfloor d \hat{J}(\xi)-V_{3}\right\rfloor d \hat{J}(\xi) \\
& =\Omega\left(\xi_{\Gamma}, V_{1}\right)+\Omega\left(\xi_{\Gamma}, V_{2}\right)-\Omega\left(\xi_{\Gamma}, V_{3}\right) \\
& =(\Omega \oplus \Omega \ominus \Omega)\left(\xi_{\Gamma \times \Gamma \times \Gamma}, V\right),
\end{aligned}
$$

where $V=\left(V_{1}, V_{2}, V_{3}\right)$ and $\xi_{\Gamma \times \Gamma \times \Gamma}=\left(\xi_{\Gamma}, \xi_{\Gamma}, \xi_{\Gamma}\right)$. The action of $G$ preserves the multiplication of $\Gamma$, i. e., it leaves $\mathscr{M}$ the graph of the multiplication invariant, and $\xi_{\Gamma \times \Gamma \times \Gamma}$ is tangent to $\mathscr{M}$. Since $\mathscr{M}$ is lagrangian, we have $V\rfloor d K=0$; that is, $K$ is locally constant on $\mathscr{M}$. Now the fibres of the map $(x, y, z) \mapsto x$ from $\mathscr{M}$ to $\Gamma_{0}$ are diffeomorphic to the $\alpha$-fibres and so are connected. Since $K(u, u, u)=0$ for all $u$, we conclude that $K \equiv 0$ on $\Gamma$ and $J(x \cdot y)=J(x)+J(y)$. 
Corollary 2. 4. Every action of a Lie group $G$ by automorphisms of a connected and $\alpha$-simply connected symplectic groupoid $\Gamma$ has a coadjoint equivariant momentum mapping $J$ with $J(x \cdot y)=J(x)+J(y)$.

Proof. It is enough to show that the action of $G$ has a momentum mapping. Fix an arbitrary $\xi \in \mathrm{g}$. Since $\left.\xi_{\Gamma}\right\rfloor \Omega$ is a closed 1 -form, there exists a local hamiltonian for $\xi_{\Gamma}$ on some connected neighborhood of each point, unique up to a constant. Take an arbitrary point $u \in \Gamma_{0}$. Then we have a local hamiltonian $f^{(u)}$ for $\xi_{\Gamma}$ on a connected neighborhood $\mathscr{U}_{u}$ in $\Gamma$ with $f^{(u)}(u)=0$. Since $\Gamma_{0}$ is lagrangian and $\xi_{\Gamma}$ is tangent to $\Gamma_{0}, f^{(u)}$ is constant on $\mathscr{U}_{u} \cap \Gamma_{0}$, that is, $f^{(u)}=0$ on $\mathscr{U}_{u} \cap \Gamma_{0}$. Thus, we have a well-defined local hamiltonian $f$ for $\xi$ on a neighborhood $N:=\bigcup\left\{\mathscr{U}_{u} \mid u \in \Gamma_{0}\right\}$ of $\Gamma_{0}$ in $\Gamma$ satisfying $f=0$ on $\Gamma_{0}$ 。 For each $x \in \Gamma$, we can get a unique local hamiltonian $f^{(x)}$ on a connected neighborhood in $\Gamma$ from $f$ on $N$ by the continuation method along a continuous curve in the $\alpha$-fibre $\alpha_{x}$. It is a standard argument that this definition is independent of the choice of a continuous curve in the $\alpha$-fibre because each $\alpha$-fibre is simply connected. Even if $x$ and $y$ belong to different fibres, $f^{(x)}$ and $f^{(y)}$ coincide on the intersection of their domains because the base function $f$ is identically zero along $\Gamma_{0}$. Thus, we have a global hamiltonian $\hat{J}(\xi)$ for $\xi_{\Gamma}$ such that $\hat{J}(\xi)=0$ on $\Gamma_{0}$; that is, we have a momentum mapping $J: \Gamma \rightarrow \mathrm{g}^{*}$ satisfying $J(u)=0$ for each $u \in \Gamma_{0}$. Applying Theorem 2.3, we complete the proof of the corollary.

Example 2.5. The cotangent bundle $T^{*} Q$ of any manifold $Q$ can be regarded as a symplectic groupoid whose symplectic structure is the cannonical one, $\alpha, \beta$ are the bundle projection $\pi$, and the multiplication is the addition in each fibre. Each fibre is diffeomorphic to $\mathbb{R}^{\mathrm{dim} Q}$ and hence simply connected. The cotangent lift of the group action of $G$ on $Q$ is a symplectic groupoid action on $T^{*} Q$. Therefore, Corollary 2. 4 applies to this example, with the following well-known result.

Corollary 2.6 ([17]). Let a Lie group $G$ act on a manifold $Q$. Then the cotangent lift of $G$ to $T^{*} Q$ has a coadjoint equivariant momentum mapping which is linear on each fibre. 
Remarks 2.7. (i) The results above are consequences of a general theory of "lifting" from Poisson manifolds to symplectic groupoids, which we hope to present in a future paper.

(ii) There is an intriguing resemblance between the results in this section and some of the material on cocyles and actions on $C^{*_{-}}$ algebras in [15]. Can this resemblance be attributed to some kind of "quantization"?

\section{§ 3. Symplectic Groupoid Actions and Reduction}

In this section, we begin by observing that the cotangent bundle of any Lie group $G$ is a symplectic groupoid. We then interpret momentum mappings $J: Q \rightarrow g^{*}$ in terms of this groupoid, so that $g^{*}$ can be replaced by the base of any symplectic groupoid.

Example 3.1. (See [3] for details.) Let $G$ be a Lie group. The natural lift to $T^{*} G$ of the action of $G$ on itself by left translations has a coadjoint equivariant momentum mapping $J_{L}: T^{*} G \rightarrow g_{-}^{*}$, and the lift of the right action has a coadjoint equivariant momentum mapping $J_{R}: T^{*} G \rightarrow \mathfrak{g}_{+}^{*}$, where $\mathfrak{g}_{ \pm}^{*}$ is the dual space $\mathfrak{g}^{*}$ with the \pm Lie-Poisson bracket (cf. [12]). $T^{*} G$ is a symplectic groupoid over $\mathfrak{g}_{+}^{*}$ with $J_{R}, J_{L}$ as the source and target maps. Using the identification of $T^{*} G$ with $\mathrm{g}^{*} \times G$ by right translations and the notations $\delta \mu \in T_{\mu} \mathrm{g}^{*} \simeq \mathrm{g}^{*}, \delta g \in T_{g} G$, $\delta g \cdot h:=T\left(R_{h}\right) \delta g$, and $\mu \cdot g:=\mu \circ A d(g)=A d(g) *(\mu)$, we may describe the symplectic groupoid structure as follows:

$$
\begin{aligned}
& \alpha(\mu, g)=\mu, \beta(\mu, g)=\mu \cdot g, \varepsilon(\mu)=(\mu, e), \\
& (\mu, g) \circ(\mu \cdot g, h)=(\mu, g h),(\mu, g)^{-1}=\left(\mu \cdot g, g^{-1}\right) ;
\end{aligned}
$$

the cotangent symplectic structure $\Omega$ in this representation is

$$
\begin{aligned}
\Omega\left((\delta \mu, \delta g),\left(\delta^{\prime} \mu, \delta^{\prime} g\right)\right)= & \left\langle\delta^{\prime} \mu, \delta g \cdot g^{-1}\right\rangle-\left\langle\delta \mu, \delta^{\prime} g \cdot g^{-1}\right\rangle \\
& -\left\langle\mu,\left[\delta g \cdot g^{-1}, \delta^{\prime} g \bullet g^{-1}\right]\right\rangle .
\end{aligned}
$$

The induced Poisson structure on $\mathrm{g}^{*}$ is the $(+)$-Lie-Poisson structure: $\left\{f_{1}, f_{2}\right\}_{0}(\mu)=\left\langle\mu,\left[\delta f_{1} / \delta \mu, \delta f_{2} / \delta \mu\right]\right\rangle$, and the hamiltonian vector field $X_{f}(\mu)$ equals $-\mu \circ a d(\delta f / \delta \mu)$, where $\delta f / \delta \mu$ is the differential of $f$ at $\mu$, considered as an element of $\mathrm{g} \simeq \mathrm{g}^{* *}$.

Let $G$ act on a symplectic manifold $M$ with a coadjoint equivariant momentum mapping $J$, so that $J: M \rightarrow g^{*}$ is a Poisson map. One 
defines an "action" of $T^{*} G \simeq g^{*} \times G$ on $M$ by $(\mu, g) \circ m:=g \circ m$ when $J(m)=\mu$ 。 This action turns out to be "symplectic" in the sense that its graph $\{((\mu, g), m,(\mu, g) \circ m)\}$ is lagrangian in $T^{*} G \oplus M \ominus M$.

The situation above is a special case of the following general notion of groupoid action. (See [2], [3], [5] or [15].) Let $\Gamma$ be a groupoid over $\Gamma_{0}$ with source map $\alpha$ and target map $\beta_{0}$. Let $M$ be a set, and let $J$ be a map from $M$ to $\Gamma_{0}$. Let $\Gamma \times M=\{(x, m) \in$ $\Gamma \times M \mid \beta(x)=J(m)\}$ and $M \underset{J}{\times} \Gamma=\{(m, x) \in M \times \Gamma \mid J(m)=\alpha(x)\}$.

Definition 3.2. A left action of $\Gamma$ on $M$ with moment $t^{2)} J$ is a mapping $(x, m) \mapsto x \circ m$ from $\Gamma \times \underset{J}{\times} M$ to $M$ satisfying:

(i) $\quad J(x \circ m)=\alpha(x)$

(ii) $(x \circ y) \circ m=x \circ(y \circ m)$

(iii) $\varepsilon(J(m)) \circ m=m$

for $x, y \in \Gamma, m \in M$. (In (ii), each side is defined if the other is, by virtue of (i).)

Right actions are defined similarly.

A space on which $\Gamma$ acts is called a $\Gamma$-space. Equivariance of maps between $\Gamma$-spaces is defined in an obvious way, compatibility of the moments being part of the definition.

Example 3. 3. (i) Any groupoid $\Gamma$ acts on itself from both sides by multiplication. The moments are $\alpha$ and $\beta$ for the left and right actions respectively.

(ii) Any groupoid $\Gamma$ acts on $\Gamma_{0}$ from both sides with moment $i d_{\Gamma_{0}}$. The left action is $x \circ u=\alpha(x)$, and the right action is $u \circ x=\beta(x)$.

2) We hope by this nomenclature to resolve in a retroactive fashion the long-standing disagreement over the English translation of Souriau's French term moment for a Poisson map to the dual to a Lie algebra. Thinking of moment as a generalization of moment cinetique (angular momentum), Abraham and Marsden [1] chose the translation "momentum", which we still believe to be most appropriate. On the other hand, Guillemin and Sternberg [6] chose the model terms "moment of momentum" and "moment of inertia" to justify the translation "moment". With the terminology introduced here, a momentum mapping becomes a special case of a moment, and so all the previous literature is made consistent.

This leaves open the question of the appropriate French translation of the English "moment" in the groupoid context: we propose that the French moment be retained, with its meaning simply extended. 
The proof of the following simple result is left to the reader.

Proposition 3.4. (i) $\alpha$ and $\beta$ are equivariant maps from $\Gamma$ to $\Gamma_{0}$ with the left or right actions of $\Gamma$ in Example 3.3.

(ii) More generally, if $(M, J)$ is any left [right] $\Gamma$-space, then the moment $J: M \rightarrow \Gamma_{0}$ is equivariant with respect to the left [right] action of $\Gamma$.

Example 3.5. Let a Lie group $G$ act on $\Gamma_{0}$ from the right, so that $\Gamma_{0} \times G=: \Gamma \simeq\{(u, g, v) \mid u \cdot g=v\}$ is a groupoid by

$$
\begin{aligned}
& \alpha(u, g, v)=u, \quad \beta(u, g, v)=v, \quad \varepsilon(u)=(u, e, u), \\
& \iota(u, g, v)=\left(v, g^{-1}, u\right), \quad(u, g, v) \cdot(v, h, w)=(u, g h, w) .
\end{aligned}
$$

This is called a transformation groupoid. (Compare Example 3.1.)

Let $M$ be a right $G$-space and $J: M \rightarrow \Gamma_{0}$ an equivariant map. Then $\Gamma$ acts on $M$ in the following way:

$$
\text { When } J(m)=\alpha(u, g, v)=u, \quad m \cdot(u, g, v)=m \cdot g \text { 。 }
$$

On the other hand, if $\Gamma_{0} \times G$ acts on $M$ with moment $J: M \rightarrow \Gamma_{0}$, then for any $m \in M, g \in G$, we have $m \bullet(J(m), g, J(m) \cdot g)$ defined. One may check that this is an action of $G$ on $M$, making $J$ equivariant. In fact, for each $G$-space $\Gamma_{0}$, there exist inverse functors between the categories ([2] [5]):

(i) $G$-spaces $M$ equipped with an equivariant map $J: M \rightarrow \Gamma_{0}$.

(ii) $\Gamma$-spaces $M$, where $\Gamma$ is the transformation groupoid $\Gamma_{0} \times G$.

We may call these spaces $\left(\Gamma_{0}, G\right)$-spaces.

Remarks 3.6. (i) If we let $\left(\Gamma_{0}, G\right)$ vary as well, we get a bigger category. (Compare the discussion in [21].)

(ii) All of the above may be generalized to the case where $G$ is a groupoid (see, [2] [5]).

Definition 3.7. Let $\Gamma$ be a symplectic groupoid and let $(M, J)$ be a $\Gamma$-space such that $M$ is a symplectic manifold. The action of $\Gamma$ on $M$ is called symplectic if the graph $\{(x, m, x \cdot m) \mid \beta(x)=J(m)\}$ of the action is lagrangian in $\Gamma \oplus M \ominus M . \quad(M, J)$ is then called a symplectic $\Gamma$-space. 
Theorem 3.8. If $(M, J)$ is a symplectic $\Gamma$-space, then the moment $J: M \rightarrow \Gamma_{0}$ is a Poisson map.

Proof. Since $\left.J^{*}\{h, f\}(m)=X_{f}(J(m))\right\rfloor d h$ and $\quad\left\{J^{*} h, J^{*} f\right\}(m)=$ $\left.X_{J^{*} f}(m)\right\rfloor d J^{*} h=T J\left(X_{J^{*} f}(m)\right\rfloor d h$, it suffices to show that $T J\left(X_{J^{*} f}\right)=X_{f}$ for each $f \in C^{\infty}\left(\Gamma_{0}\right)$. So consider a tangent vector of the form $\left(X_{\beta^{n} f},-X_{J^{*} f}, 0\right)$ in $\Gamma \times M \times M$ and tangent vector $(\delta x, \delta m, \delta x \circ m+x \circ \delta m)$ to the graph $\mathscr{A}$ of the action. We have $T \beta(\delta x)=T J(\delta m)$, and hence

$$
\begin{aligned}
& \Omega_{\Gamma \oplus M \ominus M}\left(\left(X_{\beta^{\natural} f},-X_{J^{\natural} f}, 0\right),(\delta x, \delta m, \delta x \circ m+x \circ \delta m)\right) \\
& \quad=\Omega_{\Gamma}\left(X_{\beta^{\natural} f}, \delta x\right)-\Omega_{M}\left(X_{J^{\natural} f}, \delta m\right) \\
& \quad=T \beta(\delta x)\rfloor d f-T J(\delta m)\rfloor d f \\
& \quad=0
\end{aligned}
$$

Thus, $\left(X_{\beta^{\prime \prime} f},-X_{J^{*} f}, 0\right) \in(T \mathscr{A})^{\perp}=T \mathscr{A}$ because $\mathscr{A}$ is lagrangian. Therefore, $T \beta\left(X_{\beta^{*} f}\right)=T J\left(-X_{J^{*} f}\right)$. But $T \beta\left(X_{\beta^{*} f}\right)=-X_{f}$ since $\beta$ is anti-Poisson, and so $T J\left(X_{J^{*} f}\right)=X_{f}$; i. e., $J$ is a Poisson map.

Example 3.9. Let a Lie group $G$ act on a symplectic manifold $M$ with a coadjoint equivariant momentum mapping $J_{0}$. We can define an action of the groupoid $T^{*} G$ over $g^{*}$ on $M$ by $(\mu, g) \circ m:=g \circ m$ if $\beta(\mu, g)=J_{0}(m)$. Since $T^{*} G$ is a transformation groupoid, it follows from Example 3.5 and the coadjoint equivariance of $J_{0}$ that the conditions of Definition 3.2 are satisfied for this action. It is already known that the coadjoint equivariance of a momentum mapping $J_{0}$ is equivalent to $J_{0}$ being a Poisson map, or to the graph $\left\{\left(J_{0}(g \circ m), g, m, g \circ m\right) \mid g \in G, m \in M\right\}$ being lagrangian in $T^{*} G \oplus M \ominus M$ (see [1] [18]). Therefore, the action of $T^{*} G$ on $M$ induced by the coadjoint equivariant momentum mapping is a symplectic action having the momentum mapping as its moment.

The moment of a symplectic groupoid action is thus a generalization of a coadjoint equivariant momentum mapping, so it is natural to try to generalize the symplectic reduction procedure from momenta to moments, i.e., from groups to groupoids. Remarkably, the definition and many properties of reduction turn out to be purely groupoid-theoretic, involving no symplectic geometry at all.

Let $\Gamma$ be a groupoid over $\Gamma_{0}$, and let $(M, J)$ be a $\Gamma$-space. For 
$u \in \Gamma_{0}$, the "isotropy group" $\Gamma_{u}:=\alpha^{-1}(u) \cap \beta^{-1}(u)$ acts on $J^{-1}(u)$, so we can form the reduced space $M_{u}:=J^{-1}(u) / \Gamma_{u}$.

Example 3. 10. With $\Gamma$ acting on $\Gamma$ from the right $(J=\beta)$, $J^{-1}(u) / \Gamma_{u}$ is naturally isomorphic to the orbit of $u$ in $\Gamma_{0}$ (by the $\operatorname{map} x \mapsto \alpha(x))$.

In the case of a transformation groupoid $\Gamma=\Gamma_{0} \times G$, the isotropy subgroup $\Gamma_{u}=\{(u, g, u) \mid u \cdot g=u\}$ is naturally isomorphic to the isotropy subgroup $G_{u}$ for the $G$-action. Reduction of a $\left(\Gamma_{0}, G\right)$-space $M$ thus gives $M_{u}=J^{-1}(u) / G_{u}$ for each $u \in \Gamma_{0}$.

We shall now explain how the larger reduced spaces of Marle [10] (see also [8]) can be obtained for general groupoid actions. Let $\Gamma$ be a groupoid over $\Gamma_{0},(M, J)$ a $\Gamma$-space. Let $M_{\Gamma}$ be the (disjoint) union $\underset{u \in \Gamma_{0}}{\cup} M_{u}$ of the reduced spaces. Then there is an obvious diagram

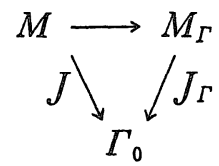

Proposition 3.11. Let $x \in \Gamma$. Then there is a well defined map $\varkappa_{(x)}(y)=y \cdot x$ from $M_{\alpha(x)}$ to $M_{\beta(x)}$ which is the same for all $z \in \alpha_{x} \cap \beta_{x}$. These maps define an action of $\Gamma$ on $M_{\Gamma}$ with moment $J_{\Gamma}$.

The action in Proposition 3.11 gives a natural trivialization of the bundle $J_{\Gamma}: M_{\Gamma} \rightarrow \Gamma_{0}$ over each orbit $\mathcal{O} \subset \Gamma_{0}$ of $\Gamma$. In other words, if we restrict the groupoid $\Gamma$ to $\Gamma_{\mathcal{O}}$ over $\mathcal{O}$, then $\Gamma_{\mathcal{O}}$ acts on $J^{-1}(\mathcal{O})$, and the reduced space $J^{-1}(\mathcal{O})_{\Gamma_{\mathcal{O}}}$ has a natural product structure $\mathcal{O} \times M^{\mathcal{O}}$, where $M^{\mathcal{O}}$ is isomorphic to $J^{-1}(u) / \Gamma_{u}$ for any $u \in \mathcal{O}$. This is our version of Marle's reduction [10].

We denote by $M / \Gamma$ the union of the $M^{\mathcal{O}}$ for all orbits $\mathcal{O}$. Note that $M / \Gamma$ is isomorphic to the quotient of $M$ by the equivalence relation $m \sim n$ if and only if $n=x \circ m$ for some $x \in \Gamma$. There is a natural map $M / \Gamma \rightarrow \Gamma_{0} / \Gamma$ which makes $M / \Gamma$ a "bundle" over the orbit space $\Gamma_{0} / \Gamma$ with fibres the reduced spaces $M_{\mathcal{O}}$.

In the case $M=\Gamma$, we have $\Gamma_{\Gamma} \simeq\left\{(u, v) \in \Gamma_{0} \mid u \sim v\right\}$, the orbit equivalence relation, while $\Gamma / \Gamma \simeq \Gamma_{0}$. For $M=\Gamma_{0}$, we have $\left(\Gamma_{0}\right)_{\Gamma}=\Gamma_{0}$, 
while $\Gamma_{0} / \Gamma$ is the orbit space, as it should be.

Now we insert some symplectic structure.

Theorem 3. 12. Let the symplectic groupoid $\Gamma$ act symplectically on $M$ with moment $J$. Assume that $u$ is a clean value of $J$ and that $J^{-1}(u) / \Gamma_{u}$ is a differentiable manifold such that the projection $h_{u}: J^{-1}(u) \rightarrow J^{-1}(u) / \Gamma_{u}$ is a submersion. Then there exists a unique symplectic structure $\Omega_{u}$ on $J^{-1}(u) / \Gamma_{u}$ such that $\kappa_{u}^{*} \Omega_{u}=\iota_{u}^{*} \Omega_{M}$, where $\iota_{u}$ denotes the inclusion map of $J^{-1}(u)$ into $M$.

Proof. As in the usual reduction theorem [13] [14], it suffices to show that the null space of the closed 2-form $\iota_{u}^{*} \Omega_{M}$ on $J^{-1}(u)$ equals the tangent space to the $\Gamma_{u}$-orbit at each point $m \in J^{-1}(u)$. Differentiating $(x, m, x \circ m) \in \mathscr{A}$ with respect to $x \in \Gamma_{u}$ at $u \in \Gamma_{0}$, we have $(\delta u, 0, \delta u \cdot m) \in T \mathscr{A}$, where $\delta u \cdot m$, the image of the tangent vector $\delta u$ by the differential with respect to $x$ the map of $(x, m) \mapsto x \cdot m$, is the general tangent vector to the orbit $\Gamma_{u} \cdot m$. Differentiating $(u, n, n) \in \mathscr{A}$ with respect to $n \in J^{-1}(u)$ at $m$, we have $(0, \delta m, \delta m) \in T \mathscr{A}$. Since $\mathscr{A}$ is lagrangian, we get $\Omega_{M}(\delta u \cdot m, \delta m)=0$, i。 e, $\delta u \cdot m \in\left[T J^{-1}(u)\right]^{\perp}$; thus the tangent space to the $\Gamma_{u}$-orbit is contained in the null space of $\ell_{u}^{*} \Omega_{M}$.

Next we show that if $\delta m$ belongs to the null space $T J^{-1}(u) \cap$ $\left[T J^{-1}(u)\right]^{\perp}$, then $\delta m \in T\left(\Gamma_{u}\right.$-orbit $)$. Since $\delta m \in\left[T J^{-1}(u)\right]^{\perp}, \delta m=X_{J^{*} f}(m)$ for some $f \in C^{\infty}\left(\Gamma_{0}\right)$. In fact, $\left.\delta m\right\rfloor \Omega$ annihilates $T J^{-1}(u)$, so it is $d h(m)$ for some $h$, which may be chosen to be the form $J^{*} f$. Note that $X_{f}(u)=0$ because $\delta m \in T J^{-1}(u)$ and $J$ is a Poisson map (Theorem 3.8). Since $\alpha$ is a Poisson map as well, $X_{\alpha^{\circ} f}$ is everywhere tangent to the $\alpha$-fibre of $x$. In addition, $X_{\alpha^{4} f}$ is tangent to all $\beta$-fibres (Theorem 1.6), so it is tangent to $\Gamma_{u}$. Now the function $F(y, m, n)=f(\alpha(y))-f(J(n))$ vanishes on $\mathscr{A}$ because of the property $J(y \cdot m)=\alpha(y)$ of the moment map $J$. Since $\mathscr{A}$ is lagrangian, $X_{F}(x, m, x \cdot m)=\left(X_{\alpha^{*} f}(x), 0, X_{J^{*} f}(x \circ m)\right)$ belongs to $T \mathscr{A}$. Thus, $X_{J^{*} f}(x \circ m)$ and in particular $\delta m=X_{J^{*} f}(m)$ is tangent to the $\Gamma_{u}$-orbit.

Examples 3.13. (i) Let $\Gamma_{0}$ be a symplectic manifold, $\Gamma=\pi\left(\Gamma_{0}\right)$. Any Poisson map $J: M \rightarrow \Gamma_{0}$ is a submersion such that the orthogonal 
spaces to the fibres are tangent to a foliation transversal to the fibres of $J$. Assume that the holonomy of this foliation gives globally defined maps between fibres. The resulting action of $\pi\left(\Gamma_{0}\right)$ on $M$ is then a symplectic action whose moment is $J$.

Now $J^{-1}(u)$ is a symplectic manifold on which the (discrete) fundamental group $\pi\left(\Gamma_{0}\right)_{u}$ acts symplectically; the reduced manifold $M_{u}$ is just the quotient $J^{-1}(u) / \pi\left(\Gamma_{0}\right)_{u}$.

If $\Gamma_{0}$ is connected, then $\Gamma$ is transitive on $\Gamma_{0}$, so the full quotient $M / \Gamma$ as well as $M_{\Gamma}$ all equal $J^{-1}(u) / \pi\left(\Gamma_{0}\right)_{u}$.

(ii) Let $\Gamma_{0}=$ a Poisson manifold $X$ with trivial Poisson structure, $\Gamma=T^{*} X$. If $J: M \rightarrow X$ is a Poisson map, then the reduced space $M_{u}$ represents a "localization" of $M$ to the point $u \in X$. For instance, if $P \rightarrow B \rightarrow X$ is a principal $G$-bundle, and $Q$ is a symplectic manifold, the phase space $M$ for a classical particle on $X$ with internal variables in the hamiltonian $G$-space $Q$ admits a Poisson mapping $J: M \rightarrow X$. (See [21].) The reduced space $M_{u}$ in this case is just the copy of the fibre $Q$ associated with $u \in X$.

(iii) If $\Gamma$ is an $\alpha$-connected symplectic groupoid over $\Gamma_{0}$, then the reduced manifolds for the action of $\Gamma$ on itself are just the symplectic leaves of $\Gamma_{0}$. (Example 3.10.) (This was observed in [13] for the case of $T^{*} G$ over $\mathfrak{g}^{*}$ as one of the first examples of reduction.)

To close this section, we show how the reduction procedure for Poisson maps can be carried out without the use of groupoids. Let $J: P^{\prime} \rightarrow P$ be a Poisson map. If $u \in P$ is any element, let $g_{u}$ be the transverse Lie algebra (cf. [19]).

Proposition 3.14. $g_{u}$ acts on $J^{-1}(u)$.

Proof. We identify $g_{u}$ with the conormal bundle to the symplectic leaf $\mathcal{O}_{u}$ through $u$, i. e., with the differentials $d f(u)$ of functions vanishing on $\mathcal{O}_{u}$. Let $f$ be such a function. Then $X_{f}$ leaves $u$ fixed, and so $X_{J^{\circ} f}$ leaves $J^{-1}(u)$ invariant since $J$ is a Poisson map.

Now we must show that the restriction to $J^{-1}(u)$ of $X_{J^{\circ} f}$ depends only on the differential of $f$ at $u$. But this follows immediately from the fact that $X_{J^{*} f}$ depends only on $d\left(J^{*} f\right)=J^{*} d f$. 
Definition 3.15. (Compare [3].) If $Y$ is a vector field on a manifold $M$, and $m \in M$, let $t_{-}(Y, m)$ and $t_{+}(Y, m)$ denote the endpoints of the interval on which the integral curve of $Y$ through $m$ is defined.

We say that the Poisson map $J: P^{\prime} \rightarrow P$ is complete if $t_{ \pm}\left(X_{f}, J(x)\right)$ $=t_{ \pm}\left(X_{J^{\circ} f}, x\right)$ for every $f \in C^{\infty}(P), x \in P^{\prime}$.

Corollary 3.16. If $G_{u}$ is the simply connected Lie group of $\mathrm{g}_{u}$, then $G_{u}$ acts on $J^{-1}(u)$ if $J$ is complete.

Proof. For $f$ vanishing on $\mathcal{O}_{u}, t_{ \pm}\left(X_{f}, u\right)= \pm \infty$. Now use standard results on transformation groups.

Theorem 3.17. If $J$ is complete and $P$ is symplectic, then $J^{-1}(u) / G_{u}$ is symplectic (under the usual assumptions of clean value, etc. to make $J^{-1}(u) / G_{u}$ a manifold).

Proof. We must show that, for $x \in J^{-1}(u), S:=\left\{X_{J^{\circ} f}(x) \mid f\right.$ vanishes on $\left.\mathcal{O}_{u}\right\}=\operatorname{Ker}\left(T_{x} J\right) \cap\left[\operatorname{Ker} T_{x} J\right]^{\perp}$. We already know from Proposition 3. 14 that $S \subset \operatorname{Ker} T_{x} J$ and from the proof of Theorem 3.12 that $\left[\operatorname{Ker} T_{x} J\right]^{\perp}=\left\{X_{J^{*} f}(x) \mid f \in C^{\infty}(P)\right\}$. Therefore, we have $S=\operatorname{Ker}\left(T_{x} J\right) \cap$ $\left[\operatorname{Ker} T_{x} J\right]^{\perp}$ 。

Remarks 3.18. (i) Notice that this reduction will coincide with that of Theorem 3.12 when $P$ is the base of a symplectic groupoid, but we don't need the groupoid to do it.

(ii) The Poisson reduction theorem of [11] can also be applied here.

\section{References}

[1] Abraham, R. and Marsden, J.E., Foundations of mechanics, 2nd ed. New York : Benjamin/ Cummings, 1978.

[2] Brown, R., Danesh-Naruie, G. and Hardy, J. P. L., Topological groupoids : II. Covering morphisms and G-spaces, Math. Nachr., 74 (1976), 143-156.

[3] Coste, A., Dazord, P. and Weinstein, A., Groupoides symplectiques, Publications $d u$ Départment de Mathématiques de l'Université de Lyon I, 2/A (1987).

[4] Drinfel'd, V. G., Hamiltonian structures on Lie groups, Lie bialgebras and the geometric meaning of the classical Yang-Baxter equations, Soviet Math. Dokl., 27(1983), 68-71. 
[5] Ehresmann, C., Catégories topologiques et catégories différentiables, Coll. de Géom. Différ. Globale, Bruxelles (1959), 137-150.

[6] Guillemin, V. and Sternberg, S., The moment map and collective motion, Ann. of Phys, 127 (1980), 220-253.

[7] Karasev, M. V., Analogues of objects of Lie group theory for nonlinear Poisson brackets. Math. USSR Izv., 28 (1987), 497-527.

[8] Kazhdan, D., Kostant, B. and Sternberg, S., Hamiltonian group actions and dynamical systems of Calogero type, Comm. Pure and Appl. Math., 31 (1978), 481-507.

[9] Lie, S., Theorie der Transformationsgruppen, Zweiter Abschnitt unter Mitwurking von Prof. Dr. Friedrich Engel, Teubner, Leibzig, 1890.

[10] Marle, C.-M., Symplectic manifolds, dynamical groups, and Hamiltonian mechanics, Diff. Geometry and relativity, Reidel (1976), 243-269.

[11] Marsden, J. and Ratiu, T., Reduction of Poisson manifolds, Lett in Math. Phys., 11 (1986), 161-169.

[12] Marsden, J., Ratiu, T. and Weinstein, A., Semidirect products and reduction in mechanics, Trans. Amer. Math. Soc., 281 (1984), 147-177.

[13] Marsden, J. and Weinstein, A., Reduction of symplectic manifolds with symmetry, Rep. Math. Phys., 5 (1974), 121-129.

[14] Meyer, K. R., Symmetries and integrals in mechanics, in Dynamical Systems, M. M. Peixoto, ed., Academic Press, New York (1973), 259-272.

[15] Renault, J., A groupoid approach to $\mathrm{C}^{*}$-algebras, Lecture Notes in Math., 793 (1980).

[16] Semenov-Tian-Shansky, M. A., Dressing transformations and Poisson group actions, Publ. RIMS, Kyoto Univ., 21 (1985), 1237-1260.

[17] Smale, S., Topology and mechanics. I, Invent. Math., 10 (1970), 305-331.

[18] Weinstein, A., Lectures on symplectic manifolds, CBMS Regional Conf. Ser. in Math., 29 (1977).

[19] - The local structure of Poisson manifolds, J. Diff. Geom., 18 (1983), 523-557.

[20] - Symplectic groupoids and Poisson manifolds, Bull. Amer. Math. Soc., 16 (1987), 101-104.

[21] - Poisson geometry of the principal series and nonlinearizable structures, $J$. Diff. Geom., 25 (1987), 55-73.

[22] - Coisotropic calculus and Poisson groupoids, Subwitted to J. Math Soc Japan. 